\title{
Aplicaciones de las Matemáticas a la Vida Diaria en los Libros de Aritmética Españoles del Siglo XVI
}

\section{Applications of Mathematics to Everyday Life in Spanish Arithmetic Books Published in the 16th Century}

\author{
María José Madrid* \\ Alexander Maz-Machado ${ }^{* *}$ \\ Carmen León-Mantero ${ }^{* * *}$ \\ Carmen López-Esteban ${ }^{* * * *}$
}

\begin{abstract}
Resumen
Este trabajo presenta un estudio sobre algunos libros de matemáticas publicados en castellano durante el siglo XVI. A lo largo de él, se han identificado y categorizado los ejemplos utilizados en estos manuales y su relación con las situaciones cotidianas de la época. Para ello se ha realizado un análisis histórico-matemático apoyado en la técnica de investigación del análisis de contenido de libros de texto, ampliamente utilizado por diversos autores en investigaciones relativas a la Historia de las Matemáticas y la Educación Matemática. Todas las obras analizadas presentan, en general, bastantes similitudes. En ellas se incluyen un elevado número de ejemplos aunque la gran mayoría de ellos están relacionados con asuntos comerciales y otras situaciones cotidianas de mercaderes y contadores.
\end{abstract}

Palabras clave: Matemáticas. Historia de las Matemáticas y la Educación Matemática. Siglo XVI. Aritmética Mercantil. Libros de Texto.

\begin{abstract}
This paper shows a study about some mathematics books written in Spanish and published during the 16th century. All the examples included in these books and their relations with daily situations from that time have been identified and categorized. In order to do that, we made an historical-mathematical analysis supported by the content analysis technique of textbooks, widely used by authors in researches related to the history of mathematics and mathematics education. In general, all the books included in this study present many similarities. The analysis has highlighted the large number of examples included in each book even though most of them are related to trade and to common situations of accountants and businessmen of that century.

\footnotetext{
* Doctora en Matemáticas por la Universidad de Salamanca (USAL). Profesora de Didáctica de la Matemática en la Universidad Pontificia de Salamanca (UPSA), Salamanca, España. Dirección postal: Facultad de Educación. C/ Henry Collet, 52-70, CP 37007, Salamanca, España. E- mail: mjmadridma @upsa.es

** Doctor en Matemáticas por la Universidad de Granada (UGR). Profesor de Didáctica de la Matemática en la Universidad de Córdoba (UCO), Córdoba, España. Dirección postal: Facultad de Ciencias de la Educación, Avenida San Alberto Magno s/n., CP 14071. Córdoba, España. E- mail: ma1mamaa@uco.es

*** Doctora en Matemáticas por la Universidad de Córdoba (UCO). Profesora de Didáctica de la Matemática en la Universidad de Córdoba (UCO), Córdoba, España. Dirección postal: Facultad de Ciencias de la Educación. Avenida San Alberto Magno s/n., CP 14071. Córdoba, España. E- mail: cmleon@ uco.es

**** Doctora en Matemáticas por la Universidad de Salamanca (USAL). Profesora de Didáctica de la Matemática en la Universidad de Salamanca (USAL), Salamanca, España. Dirección postal: Facultad de Educación. Paseo de Canalejas, 169, CP 37008, Salamanca, España. E- mail: lopezc@usal.es
} 
Keywords: Mathematics. History of Mathematics and Mathematics Education. Sixteenth Century. Mercantile Arithmetic. Textbook.

\section{Introducción}

La investigación en Historia de la Educación Matemática permite descubrir y sacar a la luz momentos, situaciones, instituciones, personajes o temas, que en un momento dado han significado un cambio de rumbo o un avance tanto para la historia de las matemáticas como para la educación matemática (MAZ-MACHADO; RICO, 2013).

Mientras la investigación en Historia de las Matemáticas permite observar puntos de vista diferentes o diversas formas de presentación de un concepto o de las ideas matemáticas (JAHNKE, 2001), para la comprensión histórica de las matemáticas existe la necesidad de otros tipos de conocimiento (GARCÍADIEGO, 2002). Se requiere conocer también los contextos sociales, económicos y científicos de la época que se pretende analizar, además de los aspectos didácticos implícitos en su transmisión.

En este sentido, la investigación en Historia de la Educación posee una finalidad educativa, pues ayuda a comprender la actividad que se ha realizado en las aulas, aporta información sobre los aspectos educativos de la época analizada e, incluso, ayuda tanto a profesores como alumnos en el proceso de enseñanza-aprendizaje. En definitiva, la investigación en Historia de la Educación Matemática amplía la comprensión proporcionada a través de la investigación en Historia de las Matemáticas profundizando en los aspectos didácticos relacionados con el proceso de enseñanza-aprendizaje de estos conceptos e ideas matemáticas.

Una de las líneas de trabajo en la investigación histórica en Educación Matemática busca en los manuales o libros de texto aquellas evidencias del interés didáctico, que manifiestan los autores, para lograr transmitir el conocimiento matemático de forma comprensible a todo aquel que tuviese deseo de aprenderlo.

Esto se debe a que, los libros para la enseñanza reflejan los hábitos y costumbres, la organización de las ideas, la actividad intelectual, las relaciones públicas de apropiación y exclusión del saber y, en muchos casos, las modas y tendencias imperantes de una época determinada, proporcionando la oportunidad de observar cómo se adquiría el saber y las relaciones con los conocimientos científicos de cada momento (MAZ-MACHADO; RICO, 2015). 
Desde el punto de vista formal de las matemáticas, los libros para la enseñanza de esta, permiten rastrear la evolución de un concepto o una idea matemática, las diferentes maneras con las que los matemáticos en el pasado se acercaron a él, las dificultades, el proceso gradual de simbolización, formalización y así sucesivamente (BRUCKHEIMER; ARCAVI, 2000). Sin embargo, los textos de matemáticas no son documentos exclusivamente formales, sino que son materiales docentes, con propósitos educativos, que se proponen transmitir unos determinados significados para la correcta comprensión de los conceptos formales que presentan (SEGOVIA; RICO, 2001).

Todas estas razones permiten que los textos históricos ayuden a reconstruir los conceptos, a contextualizarlos y a conocer sus diversos acercamientos, a interrogarse sobre la validez de las formas de argumentar vigentes en otras épocas y a buscar los fundamentos de las formas actuales. Además, estos textos informan sobre aspectos pedagógicos: las formas de organizar y presentar el contenido, sus representaciones, las situaciones, problemas y ejercicios utilizados para explicar mejor los conceptos y métodos matemáticos (GÓMEZ, 2001).

A nivel internacional son frecuentes los trabajos en los que el estudio de los manuales antiguos de matemáticas es el eje central de la investigación (GLAESER, 1981; SCHUBRING, 1987, 1988). También en España, en esta línea se han realizado diversos estudios centrados en manuales escolares de matemáticas de diversos siglos. Por ejemplo sobre la fenomenología utilizada en los manuales de matemáticas de los siglos XVIII y XIX para los números negativos (MAZ; RICO, 2009), sobre la implantación del sistema métrico decimal en las islas Canarias durante el siglo XIX (PICADO; RICO; GÓMEZ, 2013), sobre la evolución del límite funcional y del concepto de continuidad en los manuales españoles de enseñanza secundaria de la segunda mitad del siglo XX (SIERRA; GONZÁLEZ; LÓPEZ, 1999, 2003), los tipos de representaciones y la fenomenología presentes en algunos manuales del siglo XVI (MAZ-MACHADO; LÓPEZ; SIERRA, 2013; MADRID; MAZ-MACHADO, 2015; MADRID; MAZ-MACHADO; LÓPEZ, 2015) o los métodos de multiplicación en matemáticas del siglo XVI (MEAVILLA; OLLER, 2014).

Sin embargo, mientras que siglos como el XIX o el XX han sido ampliamente analizados, en el caso del siglo XVI, no son numerosos los estudios realizados sobre libros de enseñanza de matemáticas pertenecientes a dicho periodo. Esto contrasta con la relevancia del texto escrito en el siglo XVI en España, pues dicho siglo supone el primer periodo de desarrollo de la imprenta, que se implantó en España a finales del siglo XV permitiendo una 
mayor difusión de las obras escritas, en un momento en el que los libros eran el único modo de plasmar y difundir la información.

Estas razones han motivado la realización de esta investigación que presenta un estudio sobre varios libros de aritmética publicados en castellano durante el siglo XVI, libros que tenían como propósito enseñar contenidos fundamentalmente aritméticos a sus lectores. Para ello se pretende realizar un análisis de estos textos que permita conocer las aplicaciones de las matemáticas a la vida diaria presentes en ellos y todo esto ubicándolos en el periodo concreto de la historia de España en el que fueron escritos.

\section{Las matemáticas mercantiles en España durante el siglo XVI}

Desde mediados del siglo XV aproximadamente hasta finales del siglo XVI, Europa experimenta un período de crecimiento. Se produce el paso de la Edad Media a los tiempos modernos, fenómeno que tiene al menos una característica principal: el dinero adquiere un papel relevante y se convierte en el motor de la economía. En España se produce un crecimiento tanto demográfico como comercial, debido, entre otras razones, a las grandes cantidades de oro y plata que entran en Europa por Sevilla a partir de los primeros años del siglo XVI. Las remesas de Indias impulsan la ya comenzada revolución económica del XVI y las actividades comerciales experimentan un auge extraordinario (PÉREZ, 2000).

Dicho desarrollo comercial potenció que un mayor número de personas necesitaran conocimientos matemáticos básicos. Este hecho cobra mayor relevancia si se tiene en cuenta que la mayoría de la población española de este siglo era analfabeta. En muchos núcleos rurales apenas tres o cuatro personas sabían leer o escribir y en las ciudades, donde las circunstancias eran más favorables, había numerosos iletrados incluso entre los artesanos especializados (LÓPEZ, 1979).

La adquisición de estos conocimientos, relacionados fundamentalmente con los negocios, se vio también favorecida por el desarrollo de la imprenta, que se introdujo en España en 1472, pues ésta hizo que proliferaran todo tipo de tratados acerca de cómo ser comerciante (BURKE, 2000). Esto favoreció que, a lo largo de este siglo, surgieran en Europa una serie de libros de aritmética con la clara intención de transmitir una matemática de carácter comercial. De hecho, desde finales del siglo XV hasta el siglo XVI, las aritméticas mercantiles son las obras de matemáticas más impresas y que llegan a un público más amplio (MALET, 2000). 
Entre los autores españoles del siglo XVI la actividad matemática se desarrolló en torno a dos líneas diferentes, como disciplina teórica de carácter formal y como base de aplicaciones prácticas en el mundo real asociadas a la economía y la técnica (LÓPEZ, 1979). En la primera, más académica, destacaron autores como Pedro Sánchez Ciruelo y Juan Martínez Silíceo. En la línea de las aplicaciones, la más destacada en España fue el cálculo mercantil, tanto es así, que esta aritmética práctica concebida como útil herramienta de cálculo para la resolución especialmente de los problemas de la aritmética comercial jugó un importante papel en el despliegue del capitalismo comercial (SALAVERT, 1994).

En la línea de las aplicaciones prácticas de las matemáticas, Salavert (1994) contabiliza un total de 43 primeras ediciones de aritmética práctica entre 1482 y 1600, 77 obras contando las distintas ediciones y un total de 35 autores. Más concretamente, en el siglo XVI en España se publican, entre otros, el "Tractado sutilissimo de Arismetica y de Geometria”, de Juan de Ortega, en 1534, la primera edición es de 1512 en Lyon (REY, 1934), el "Sumario breve de la practica de arithmetica de todo el curso del arte mercantivol", de Juan Andrés, en 1515, la "Practica mercantivol", de Joan Ventallol, en 1521, el "Tratado de cuentas", de Diego del Castillo, en 1522, el "Compendio de los números y proporciones", de Pedro Melero, en 1535, el "Arte breve y muy provechoso de cuenta castellana y Arismetica", de Juan Gutiérrez de Gualda, en 1539, el "Libro primero de arithmetica algebraica", de Marco Aurel, en 1552, o la "Arithmetica practica y especulativa", de Juan Pérez de Moya, en 1562.

En la mayoría de manuales orientados al cálculo mercantil publicados a lo largo de este siglo se presentan contenidos muy semejantes. Estos se inician con la descripción del sistema de numeración y luego se presentan las cuatro operaciones aritméticas básicas, las fracciones y sus operaciones. Prosiguen con la regla de tres, las progresiones y algunas incluyen la extracción de raíces cuadradas y cúbicas (PARADÍS; MALET, 1989).

Incluso será dentro de esta vertiente práctica donde se incluyan los avances matemáticos que ya se reflejaban en otros países, por ejemplo el alemán Marco Aurel escribió, en 1552, su libro "Arithmetica algebratica", considerado el primer libro impreso escrito en español en el que se trata el álgebra. A partir de este momento, otros autores como Pérez de Moya o Rocha siguen a Aurel, incluyendo en sus obras capítulos en los que se trata el álgebra.

Además, estas aritméticas mercantiles destacan por contener un gran número de problemas especialmente orientados para servir como ejemplo a los mercaderes en las situaciones semejantes y frecuentes que ocurrían en el mundo del comercio de la época. Por eso la producción de estas obras matemáticas quedó ligada a las ciudades más ricas y 
económicamente desarrolladas, donde trabajaban los autores por la demanda de sus conocimientos (PARADÍS; MALET, 1989).

Además, muchos de estos libros de aritmética escritos en lengua romance pese a no ser propiamente libros escolares, tuvieron influencia explícita en los primeros textos de enseñanza y parece razonable suponer que en ellos aprendían los maestros de las escuelas de niños durante el Antiguo Régimen (SIERRA; RICO; GÓMEZ, 1997). De hecho, Caunedo (2009) afirma que estos manuales de aritmética fueron publicados para su uso como textos escolares en los que los ejemplos y problemas están asociados a situaciones concretas en las que los mercaderes podrían verse involucrados.

Este fenómeno de tratar de conectar las matemáticas con las necesidades de la sociedad que como se ha visto ya ocurrió en el pasado, es uno de los aspectos que en la actualidad se promueven en los sistemas educativos en relación con las matemáticas y su carácter interdisciplinario. Fomentándose la importancia de conectar las matemáticas con otros conocimientos, con otros elementos del currículo y con el mundo diario, aspectos que el National Council of Teacher of Mathematics (NCTM) viene proponiendo desde los años 80 (NCTM, 1989, p. 32) "opportunities to make connections so that students can use mathematics in their daily lives".

Si bien en el pasado esta conexión entre las matemáticas y la vida diaria obedecía más a una necesidad practica dada lo restringida que era la población que podía tener acceso a estos conocimientos, mientras que en la actualidad estas conexiones que se fomentan están más relacionadas con el desarrollo de las competencias matemáticas básicas que toda persona debe tener en una sociedad en la que los conocimientos matemáticos no solo están a su alcance, sino que son obligatorios en los sistemas educativos.

Sin embargo, esta idea que puede parecer actual ya estaba presente en la mayoría de libros de matemáticas de épocas pasadas. Por tal razón se ha planteado como objetivo de esta investigación analizar algunos libros de matemáticas publicados en España en el siglo XVI con el fin de identificar qué tipo de conexiones se realizaban con la vida diaria de entonces. Recientemente, siguiendo esta misma idea de analizar históricamente problemas sobre aspectos determinados y puntuales, se ha realizado un estudio sobre problemas relacionados con las decisiones acerca de la ubicación física de un conjunto de instalaciones (recursos) para minimizar los costos de localización desde una perspectiva histórica (BRUNO; GENOVESE; IMPROTA, 2014). Esto muestra el interés y actualidad de este tipo de estudios. 


\section{Metodología}

La investigación realizada es cualitativa, exploratoria, descriptiva, muestral y ex post facto. El modelo de investigación a través del cual se aborda el problema es el histórico, basándose en el análisis de libros de texto antiguos de matemáticas, en este caso en los libros de texto de matemáticas del siglo XVI publicados en España y en los contenidos matemáticos presentes en ellos con atención especial a la aritmética. En el análisis se ha puesto el foco en uno de los aspectos que Sierra, Rico y Gómez (1997) señalan como adecuado para el estudio histórico de los libros: las aplicaciones a las que se orientan los conocimientos matemáticos. Para categorizar estas aplicaciones a las que los autores orientan los conocimientos matemáticos se tuvo en cuenta la clasificación propuesta por Maz-Machado, Rico y Sierra (2013) sin ser esta excluyente de otras posibles categorías que pudieran aparecer posteriormente al realizar el análisis de las obras.

Como técnica de investigación se utilizó el análisis de contenido, porque es uno de los métodos empleados frecuentemente para la investigación en Educación Matemática (FERNÁNDEZ-CANO; RICO, 1992), siguiendo las pautas propuestas por Maz (2009) y utilizadas en otros estudios históricos de corte similar (MAZ; RICO, 2009; MAZMACHADO; RICO, 2013; MAZ-MACHADO; LÓPEZ; SIERRA, 2013).

Se definieron como unidades de análisis los enunciados de todos los problemas presentes en cada uno de los manuales estudiados. Para ello se leyeron y analizaron todos los ejemplos utilizados en los libros y, posteriormente, se categorizaron.

Para escoger la muestra de estudio se definieron como criterios de selección que los libros estuvieran escritos en castellano, que su primera impresión se realizara durante el siglo XVI, que su titulo incluyese la denominación de aritmética y que las obras estuviesen disponibles para su estudio (lo que hizo que la muestra fuera intencional y por conveniencia). Finalmente, se escogieron las siguientes nueve obras:

- "Conpusicion de la arte de la arismetica y juntamente de geometría”, escrita por Juan de Ortega y publicada en 1512, en Lyon, en Casa de Maistro Nicolau de Benedictis. Se ha analizado la primera edición de la obra.

- "Sumario breve de la practica de la arithmetica", su autor es Juan Andrés y fue publicada en 1515, en Valencia, por Juan Joffre. Se trata de la primera edición de la obra. 
- "Suma de Arihtmetica Practica y de todas Mercaderias con la horden de contadores", cuyo autor es Gaspar de Texeda. Se trata de la primera y única edición conocida de la obra, publicada en 1546, en Valladolid, en la Oficina de Francisco Fernández de Córdoba.

- “Arithmetica Practica”, su autor es Juan de Yciar. La edición analizada es la primera y única edición conocida de la obra que fue publicada en 1549, en Zaragoza, en Casa de Pedro Bernuz.

- "Libro Primero de Arithmetica Algebratica", escrito por Marco Aurel. La obra consta de una única edición conocida que fue publicada en 1552, en Valencia, en Casa de Ioan de Mey Flandro.

- “Arithmetica practica, y specvlatiua”, elaborada por Juan Perez de Moya. Se ha analizado la primera edición de la obra publicada en 1562, en Salamanca, por Mathias Gast.

- “Arte Breve y muy provechoso de quenta castellana y Arithmetica”, su autor es Juan Gutierrez. Fue publicada en 1564, en Zaragoza, en Casa de Pedro Bernuz. Algunos autores hablan de una primera edición impresa en Toledo, en 1531 (SALAVERT, 1990; SMITH, 1908), mientras que otros consideran la edición de 1539 como la primera (AUSEJO, 2015; PICATOSTE, 1891).

- "Arithmetica”, elaborada por Antich Rocha. Esta obra fue publicada en 1564, en Barcelona, en Casa de Claudio Bornat a la Águila Fuerte. Se ha analizado la primera edición de la obra.

- "Libro de arithmetica especvlativa, y práctica, intitvlado, el Dorado Contador, contiene la fineza y reglas de contar oro y plata, y los Aneajes de Flandes". Su autor es Miguel Geronimo de Santa Cruz; fue publicada en 1625, en Madrid, por Viuda de Alonso Martín. Su primera edición corresponde a 1594, sin embargo la utilizada en este estudio es la de 1625 .

La Figura 1 muestra como ejemplo las portadas de dos de las obras analizadas. 

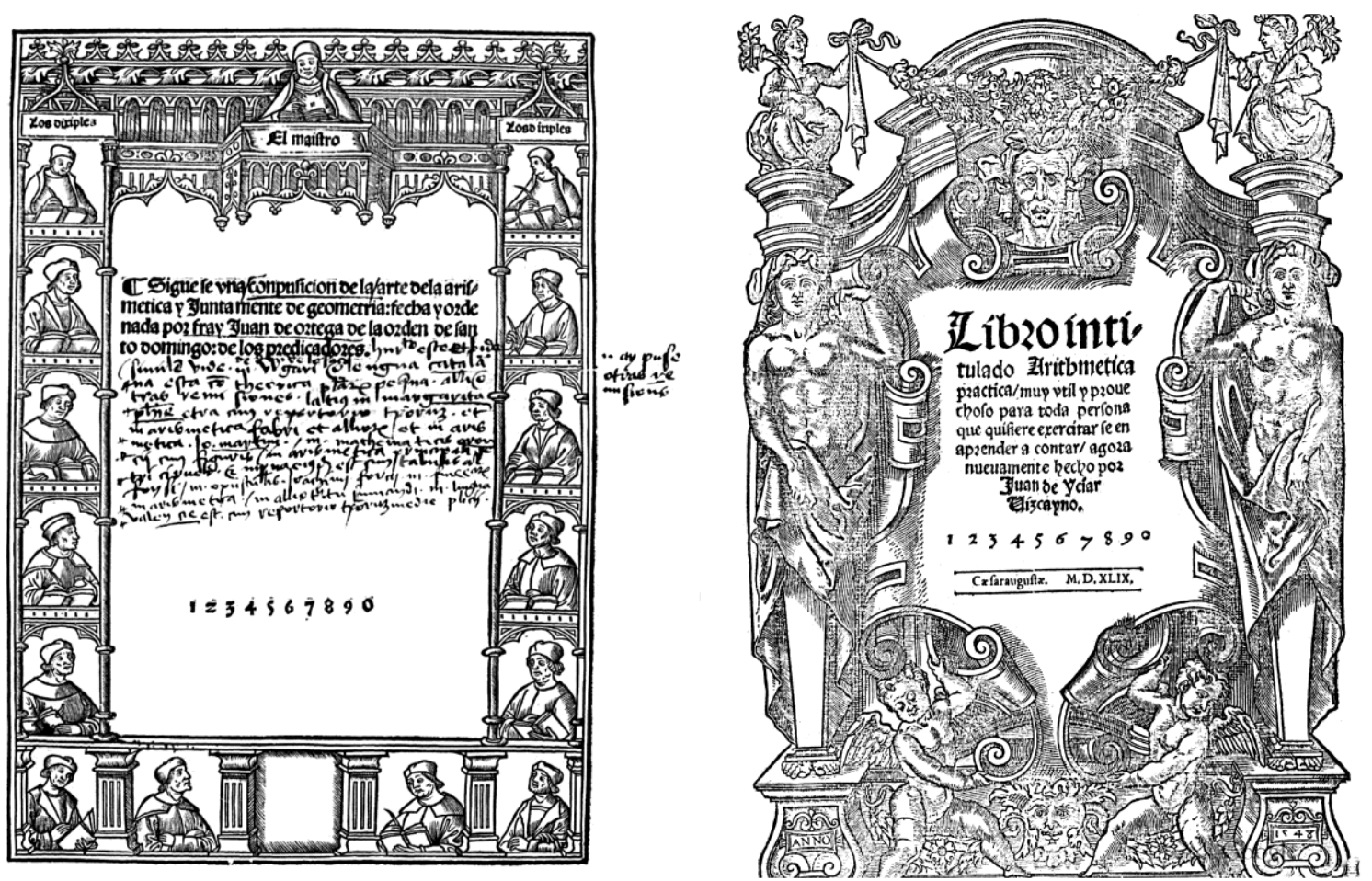

Figura 1 - Portadas de la obra de Juan de Ortega y de la de Juan de Yciar Fuente: libros de Juan de Ortega (1512) y Juan de Yciar (1549), respectivamente

\section{Resultados}

Estas obras estaban dirigidas de manera particular a los comerciantes y por ello incorporaban un gran número de problemas directamente relacionados con las necesidades de los mercaderes en el siglo XVI: compra y venta de mercancías, portes, intereses, descuentos, reducción de monedas, cambio de monedas etc. El propósito no sólo era que aprendieran las reglas básicas de la aritmética, sino que encontraran ejemplos prácticos relacionados con la actividad comercial que pudieran servir como modelo a situaciones semejantes.

Las conexiones que los autores hacen entre las matemáticas y la realidad se clasificaron, basándose en la propuesta de Maz-Machado, López y Sierra (2013) sin ser esta excluyente de otros tipos que pudiesen aparecer, en cuatro grandes grupos subdivididos después, a su vez, en categorías. La figura 2 presenta el esquema de situaciones categorizadas tras el análisis. 


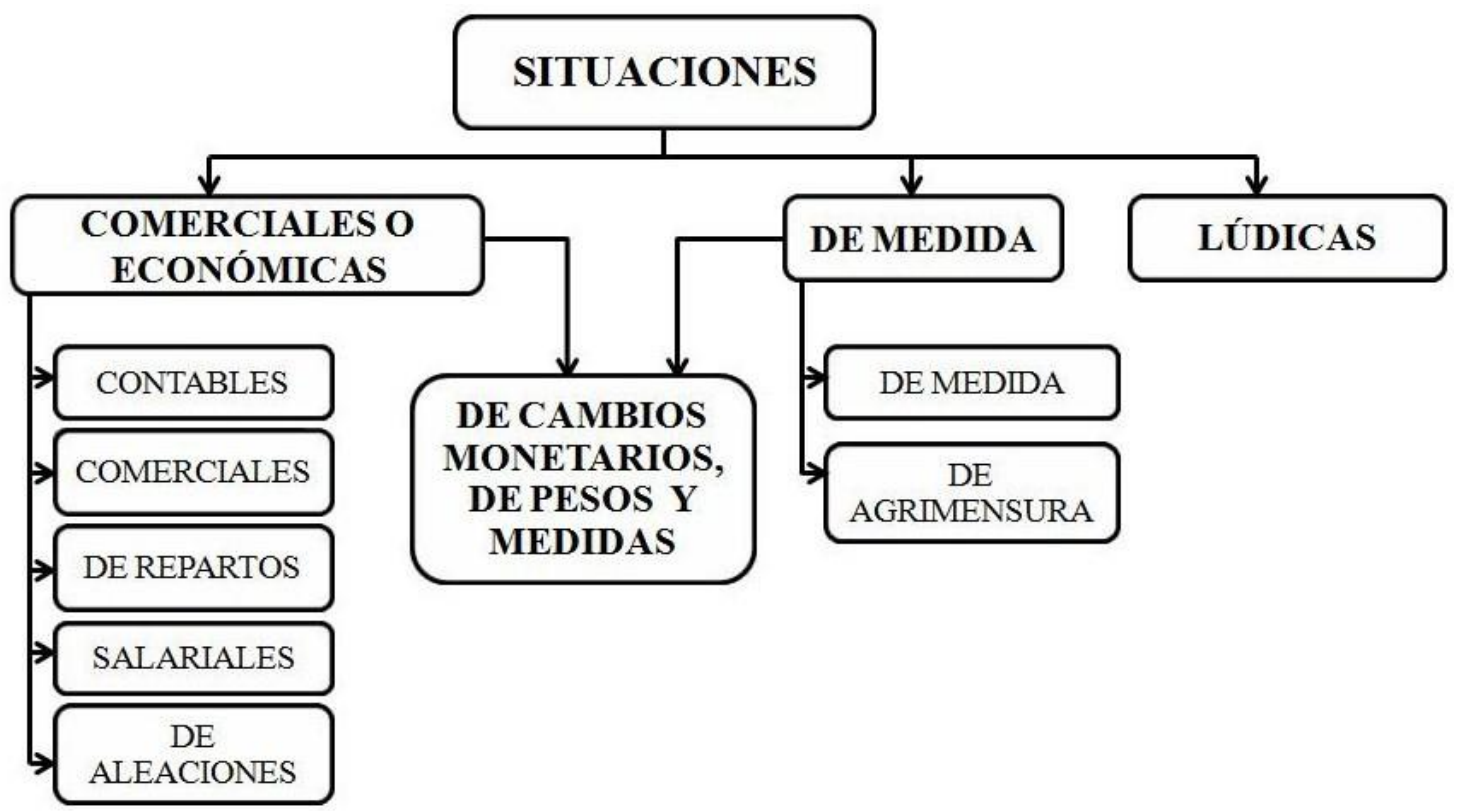

Figura 2 - Esquema de las situaciones incluidas en las obras Fuente: elaborada por los autores

A continuación se presenta cada situación con un ejemplo:

- Situaciones relacionadas con la actividad comercial y los negocios. Se han incluido en esta categoría las siguientes:

a. Situaciones contables: se presentan asociadas a problemas en los que hay que determinar una ganancia o una pérdida económica (Figura 3).

\section{EArticulo primero fi ma carga oe pebze cofto.6 c.ouc.oes inando poz quanto fe oeue vender poz ganar a razon oe.10. por ciento efta pregunts $\gamma$ las femejantes fe oenen fazer poz}

Figura 3 - Ejemplo de situación contable Fuente: Andrés (1515, p. 83)

b. Situaciones comerciales: se utilizan cuando son planteados contextos de compras y ventas de objetos, animales etc. (Figura 4).

29 2ิن Tres ciudadanos quieren cŏprar vn huerto, y ningūo ticne tanto $\bar{q}$ folo lo pueda pagar. Dize el $\mathrm{p}^{\circ}$ a los otros dos. Dad me los $\frac{2}{3}$ de lo que teneys, y conloğ yo tengo pagare folo el huerto. Dize el fegundo a los otros dos, que le den los $\frac{3}{5}$. El tercero dize alos otros dos $\bar{q}$ le den los $\frac{4}{7}$, y cada vno por li pagaria el huerto. Demando, quanto tenia cada vno, y quanto valia el huerto. Bufca vn n ${ }^{\circ}$ (como en la pafs

Figura 4 - Ejemplo de situaciones comerciales Fuente: Aurel (1552, p. 28) 
c. Situaciones de repartos: Se hallan cuando la situación planteada requiere de la distribución equitativa de objetos o ganancias, o cuando se requiere de la aplicación de la regla de compañía para distribuir la rentabilidad de un depósito o negocio (Figura 5).

\section{Tres höbres hizieron compañia, el primero pa} fo. 20. libras,y eftuuo en la compañia. 6. mefes, el fegundo pufo.16. libras,y eftuuo en la compañia.8. mefes, el tercero pufo.30. libras, y eftuuo en la com pañia. Io. mefes:en fin defte tiempo ganaron. 300. libras, demarido quanto verna a cada vno ? Haras

Figura 5 - Ejemplo de la regla de compañía Fuente: Rocha $(1564$, p. 146)

d. Situaciones salariales: en general se utilizan para aplicar reglas de tres o de falsa posición con salarios, alquileres, rentas y otros pagos como excusa para su uso (Figura 6).

\section{Vn feñor da a vn criado po.6.mefes.13. ducados y vna capa , defpues de los dos mefes va fe el cria- do con fu capa, y el feñor queda có.13.ducados,de- mando quáto auia de valer la capa,para que el mo ço fueffe fatisfecho,diras đfta manera, fi por.4.me}

Figura 6 - Ejemplo de situaciones de salarios Fuente: Rocha (1564, p.112)

e. Situaciones de aleaciones: se presentan ejemplos de aleaciones y ligaduras entre metales según diversas especificaciones dadas (Figura 7).

\section{If En cierta moneda de villon ecban|; , |marcos oe cobzey vno de plata peegunto a como/o quantosoinerostiene de ley opera comoarriba, y oigo que tiene a $\frac{3}{10}$ oinero oe leq oe plata fina que esa $\frac{1}{40}$ oe leq a répecto oe $\mid$ × $2 \mid$ oineros.}
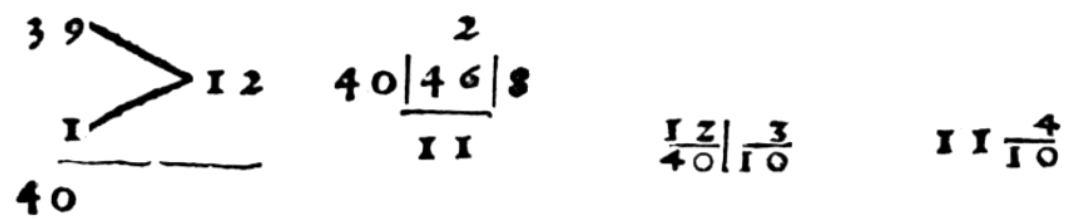

Figura 7 - Ejemplo de aleación

Fuente: Texeda (1546, p. 45)

En muchos casos estas situaciones se presentan de forma conjunta, por ejemplo el cálculo de ganancias o de perdidas en una venta o un reparto, o el cálculo del coste de una pieza de oro o plata.

- Situaciones relacionadas con la medida: 
a. Situaciones de medida: se incluyen aquellos problemas en los que se hallan longitudes de objetos, distancias o recorridos; también se han considerado aquellos relacionados con la medida del tiempo (Figura 8).

\section{-70 Eemploquarto oe medida.}
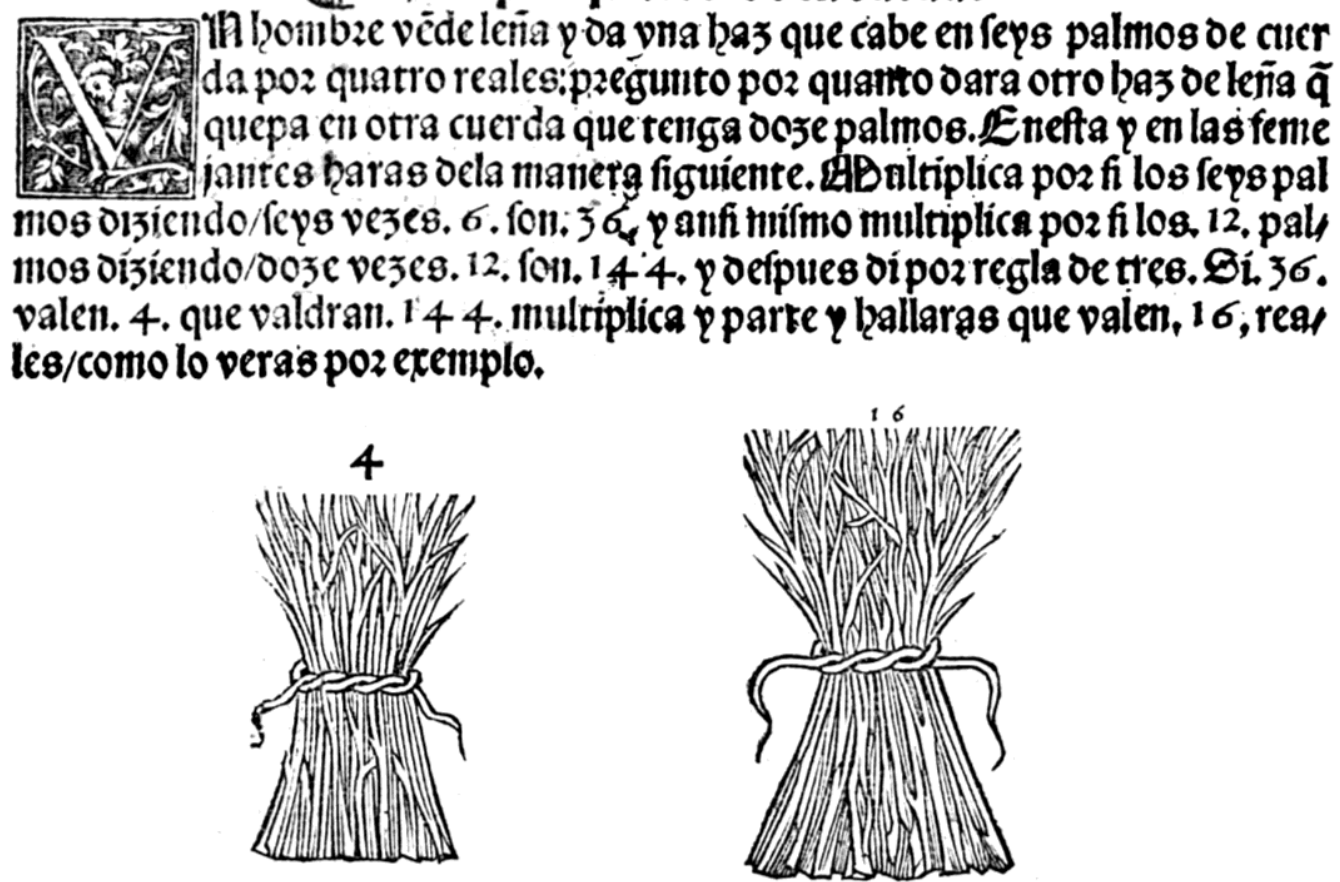

Figura 8 - Ejemplo de situaciones de medida Fuente: Yciar (1549, p. 45)

b. Situaciones de agrimensura: los autores recurren a la geometría cuando quieren aplicar conceptos y fórmulas a terrenos que tienen teóricamente formas poligonales o geométricas (Figura 9).

TEnremploregundo.

TEevna tierra quadradaqutiene pozcada quadrăgulo.8.canas la āl tierra tiene yn pafladizo oela vna égna ala orra : oemădo que quãtas canas tiene oelargo el oicbo paffadizo. C Karas anfi las canae ätiené las.z.fazes:multiplicalas vnas pos lasotras oiziēdo.8.vezes.8.fo.64.' Y oerpues oobla eftos.64.ymötară. $1 z 8$. oclos quales quita la raiz qua

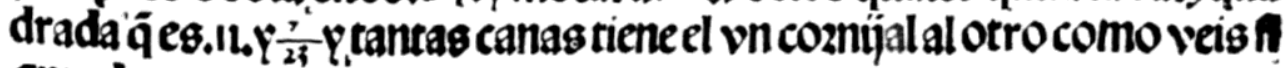
gurado.

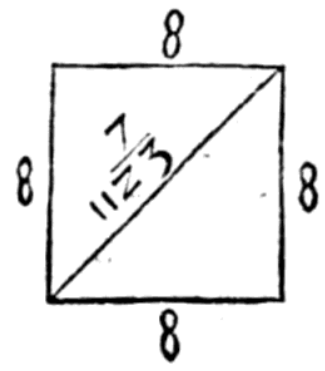

Figura 9 - Ejemplo de situación de agrimensura.

Fuente: Ortega (1512, p. 193) 
Además, sin formar parte de estas dos categorías, pero sí estrechamente relacionadas con ambas se incluyen las siguientes situaciones:

- Situaciones de cambios monetarios, de pesos y medidas: Se plantean situaciones de equivalencias entre monedas de diversas regiones y países, o en las que se debe encontrar la equivalencia entre determinadas medidas o pesos utilizados en regiones geográficas diferentes (Figura 10).

II $Y$ es afsi de marauedis reales como fi dixeffemos: ochenta ducados quá tas mil marauedis fon:quitaras la meytad, y quedaran en quarenta:y đlos

Figura 10 - Ejemplo de cambio monetario

Fuente: Gutiérrez (1564)

Finalmente, forman parte también de varias obras:

- Situaciones lúdicas: Se incluyen problemas referidos a juegos o a matemáticas recreativas como pequeñas adivinanzas (Figura 11).

\section{Thefta mefma manera fe bara fi queremos faber tres dados que vno ecbal: quantos puntos falieron en cada vno: p pongo que boúcfien ecb̨ado los tres

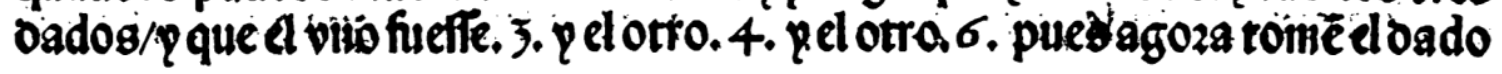

\section{Figura 11 - Ejemplo de matemáticas recreativas}

Fuente: Yciar (1549, p. 41)

Se han excluido de esta clasificación aquellos ejemplos que son sólo de naturaleza matemática, ya sea aritmética, algebraica o geométrica, sin conexión con alguna situación de la vida diaria y que se encuentran presentes en la mayoría de los libros. Por ejemplo ejercicios aritméticos como los de la Figura 12, geométricos como la Figura 13 o algebraicos como la Figura 14.

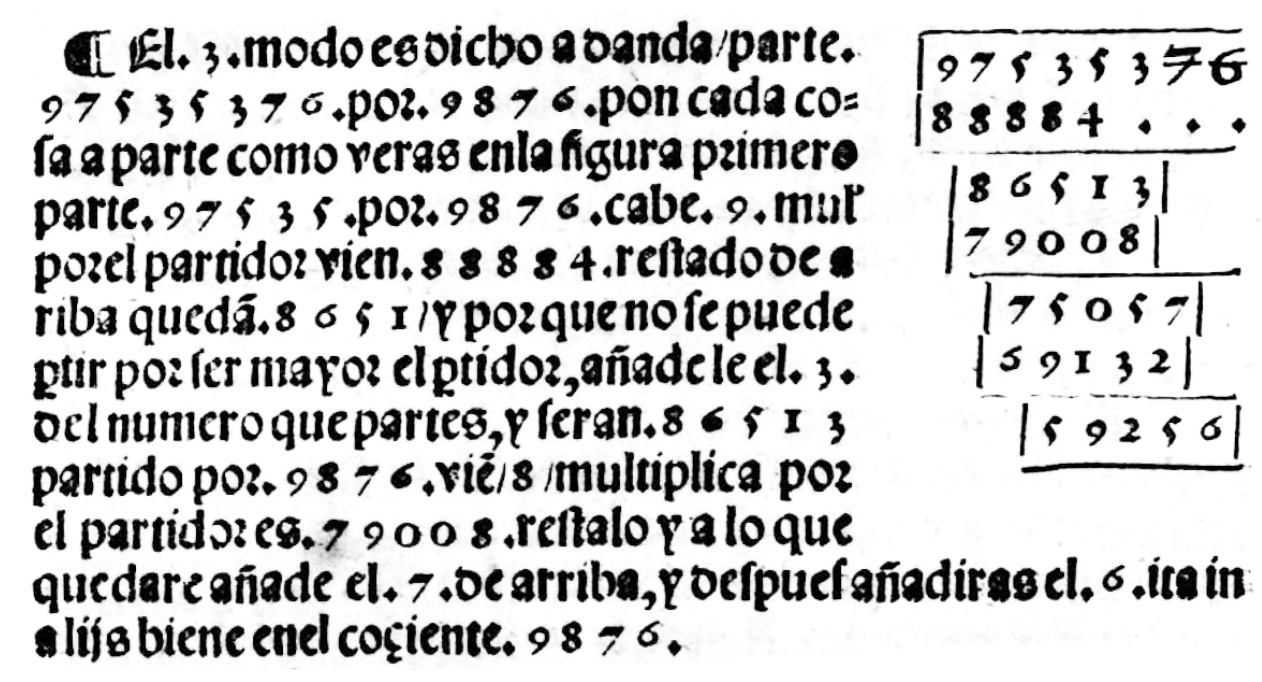

Figura 12 - Realización de una división sin contextualizar Fuente: Texeda (1546, p. 19) 


\section{TEs en cículo que tiene poz oiametro fiete eftados/pzegunto quanto tiene

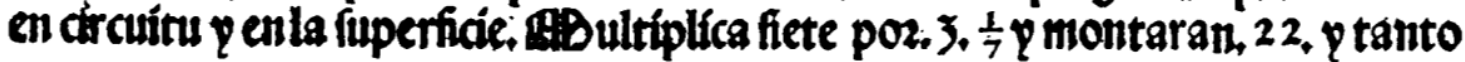

Figura 13 - Situación geométrica Fuente: Yciar (1549, p. 43)

3 aे Quando $8 u+4 Q$ fon ygual a $5 u+22 Q$, quita $4 Q$ de cada parte, y vernă $8 u$ ygual a $5 u+18 Q$. Agora quita tam bien $52 e$ de cada parte, y quedaran $; 2 e$ ygual a $18 Q$ : vale la $2 e 6 Q$.

Figura 14 - Situación puramente algebraica Fuente: Aurel (1552, p. 80)

En el Cuadro 1 se presenta la forma en que se distribuyen el tipo de situaciones presentes en los libros analizados.

\begin{tabular}{|l|c|c|c|c|c|c|c|c|c|}
\hline Autores & $\begin{array}{c}\text { Juan de } \\
\text { Ortega } \\
1512\end{array}$ & $\begin{array}{c}\text { Juan } \\
\text { Andrés } \\
1515\end{array}$ & $\begin{array}{c}\text { Gaspar } \\
\text { de } \\
\text { Texada } \\
1546\end{array}$ & $\begin{array}{c}\text { Juan } \\
\text { de } \\
\text { Yciar } \\
1549\end{array}$ & $\begin{array}{c}\text { Marco } \\
\text { Aurel } \\
1552\end{array}$ & $\begin{array}{c}\text { Juan } \\
\text { Pérez de } \\
\text { Moya } \\
1562\end{array}$ & $\begin{array}{c}\text { Juan } \\
\text { Gutiérrez } \\
1564\end{array}$ & $\begin{array}{c}\text { Antich } \\
\text { Rocha } \\
1564\end{array}$ & $\begin{array}{c}\text { Miguel } \\
\text { Gerónimo } \\
\text { de Santa } \\
\text { Cruz } \\
1625\end{array}$ \\
\hline Contable & $\mathrm{X}$ & $\mathrm{X}$ & $\mathrm{X}$ & $\mathrm{X}$ & $\mathrm{X}$ & $\mathrm{X}$ & & $\mathrm{X}$ & $\mathrm{X}$ \\
\hline Comercial & $\mathrm{X}$ & $\mathrm{X}$ & $\mathrm{X}$ & $\mathrm{X}$ & $\mathrm{X}$ & $\mathrm{X}$ & $\mathrm{X}$ & $\mathrm{X}$ & $\mathrm{X}$ \\
\hline Repartos & $\mathrm{X}$ & $\mathrm{X}$ & $\mathrm{X}$ & $\mathrm{X}$ & $\mathrm{X}$ & $\mathrm{X}$ & $\mathrm{X}$ & $\mathrm{X}$ & $\mathrm{X}$ \\
\hline Pagos & $\mathrm{X}$ & $\mathrm{X}$ & $\mathrm{X}$ & & $\mathrm{X}$ & $\mathrm{X}$ & & $\mathrm{X}$ & $\mathrm{X}$ \\
\hline Aleaciones & $\mathrm{X}$ & $\mathrm{X}$ & $\mathrm{X}$ & & $\mathrm{X}$ & $\mathrm{X}$ & & $\mathrm{X}$ & $\mathrm{X}$ \\
\hline Medida & $\mathrm{X}$ & $\mathrm{X}$ & $\mathrm{X}$ & $\mathrm{X}$ & $\mathrm{X}$ & $\mathrm{X}$ & $\mathrm{X}$ & $\mathrm{X}$ & \\
\hline Agrimensura & $\mathrm{X}$ & & $\mathrm{X}$ & $\mathrm{X}$ & & $\mathrm{X}$ & & & $\mathrm{X}$ \\
\hline Equivalencias & $\mathrm{X}$ & $\mathrm{X}$ & $\mathrm{X}$ & $\mathrm{X}$ & $\mathrm{X}$ & $\mathrm{X}$ & $\mathrm{X}$ & $\mathrm{X}$ & $\mathrm{X}$ \\
\hline Lúdicas & $\mathrm{X}$ & $\mathrm{X}$ & $\mathrm{X}$ & $\mathrm{X}$ & $\mathrm{X}$ & $\mathrm{X}$ & & $\mathrm{X}$ & $\mathrm{X}$ \\
\hline
\end{tabular}

Cuadro 1 - Cuadro resumen de las situaciones incluidas en cada libro

Fuente: elaborado por los autores

El Cuadro 1 muestra la gran importancia que conceden los autores a las relaciones comerciales de compra y venta de objetos o ganado, a los repartos de posesiones y a las equivalencias bien de monedas, pesos o medidas. En un periodo como el siglo XVI español, en el que las distintas regiones poseían diferentes monedas y medidas, resulta comprensible la importancia del cálculo correcto y con soltura de conversiones para evitar engaños en las compras, ventas, repartos etc.

Son también comunes a la mayoría de las obras, las referencias a ganancias y pérdidas económicas, a situaciones de medida, a juegos u otros aspectos recreativos y los ejemplos sobre aleaciones de oro y plata o sobre pagos. En definitiva, salvo las situaciones de agrimensura, el resto forman parte de la gran mayoría de ellos. 
Finalmente, añadir que las obras de Pérez de Moya y Gaspar de Texeda presentan la mayor variedad y riqueza de contextos. La obra de Juan Gutiérrez probablemente por su brevedad, incluye el menor número de situaciones.

\section{Conclusiones}

El auge del comercio durante el siglo XVI, impulsado por los descubrimientos geográficos y con ellos las nuevas mercancías que se compraban y distribuían por diferentes regiones y países europeos, hacía necesario tener al menos unos conocimientos matemáticos asociados a los cambios de monedas, pesos y medidas. Los autores de libros de matemáticas procuraron dar respuesta a esta necesidad al incorporar estos temas en sus obras, lo cual pone de manifiesto cómo las matemáticas tienen aplicaciones en el mundo cotidiano trascendiendo de lo puramente matemático.

Las obras analizadas pese a no presentar grandes avances matemáticos, sí poseen un valor instrumental. Los autores se preocuparon por facilitar las operaciones financieras y comerciales comunes en la época, por eso incluyen una serie de ejercicios que buscaban servir como modelo para posibles situaciones que se dieran en el comercio. Por tanto, este estudio ha puesto en evidencia que los autores del siglo XVI tenían en cuenta las necesidades cotidianas de su sociedad y pretendían acercar las matemáticas a dichas necesidades. Por eso, entre los numerosos ejemplos encontrados en las obras la mayor parte tienen relación de un modo u otro con las transacciones comerciales.

En todas las obras analizadas aparecen ejemplos de compra y venta de objetos, repartos realizando la regla de la compañía, equivalencias tanto entre monedas como con pesos y medidas, ganancias y pérdidas de dinero, pagos de salarios o rentas, situaciones de medida, lúdicas o ejemplos sobre aleaciones de oro y plata. Finalmente, aparecen también, aunque en un menor número de libros, situaciones sobre cálculos de dimensiones de terrenos.

De esta forma, la idea de conectar las matemáticas que se enseñan con el mundo real, para así darles un sentido más allá del aspecto formal no solo está presente en los manuales, currículos escolares y en prácticamente toda la comunidad de educadores matemáticos actuales, si no que ya se trabajaba y se consideraba relevante en el pasado.

El siguiente paso en esta línea de investigación deberá centrarse en comparar estos resultados con los tipos de situaciones que se presentan en libros de matemáticas de la época pero escritos en otros países, como por ejemplo la Summa, de Luca Pacioli, publicada en Italia en 1494, o la Arithmetica integra, de Stifel, publicada en Alemania en 1544, o incluso escritos 
por autores españoles de la época pero en otros idiomas como las obras de Pedro Sánchez Ciruelo o de Juan Martínez Silíceo.

\section{Agradecimientos}

Este artículo se ha realizado dentro del proyecto de investigación del Plan I+D+I del Ministerio de Economía y Competitividad de España EDU2011-27168.

\section{Referencias}

ANDRÉS, J. Sumario breve de la practica de la arithmetica. 1. ed. Valencia: Juan Joffre, 1515. 144 p.

AUREL, M. Libro Primero de Arithmetica Algebratica. 1. ed. Valencia: casa de Ioan de Mey Flandro, 1552. $140 \mathrm{p}$.

AUSEJO, E. New Perspectives on Commercial Arithmetic in Renaissance Spain. In: ROWE, D. E.; HORNG, W. (Ed.). A Delicate Balance: Global Perspectives on Innovation and Tradition in the History of Mathematics. Cham: Springer, 2015, p. 181-207.

BRUNO, G.; GENOVESE, A.; IMPROTA, G. An historical perspective on location problems. BSHM Bulletin: Journal of the British Society for the History of Mathematics, London, v. 29, n. 2, p. 83-97, 2014.

BRUCKHEIMER, M.; ARCAVI, A. Mathematics and its history: an educational partnership. In: KATZ, V. J. (Ed.). Using history to teach mathematics: an international perspective. Washington: MAA, 2000, p. 135-146.

BURKE, P. A social history of knowledge. 1. ed. Cambridge: Blackwell Publishers, 2000. 268 p.

CAUNEDO, B. Un manual de aritmética mercantil de Mosen Juan de Andrés. Pecnia, León, v. 8, p. 71-96, 2009.

FERNÁNDEZ-CANO, A.; RICO, L. Prensa y educación matemática. 1. ed. Madrid: Síntesis, 1992. $239 \mathrm{p}$.

GARCÍADIEGO, A. R. History of mathematics, an intuitive approach. Humanistic Mathematics Network Journal, Claremont, v. 26, p. 6-11, 2002.

GLAESER, G. Epistémologie des nombres relatifs. Recherches en Didactique des Mathématiques, Grenoble, v. 2, n. 39, p. 303-346, 1981.

GÓMEZ, B. La justificación de la regla de los signos en los libros de texto: ¿Por qué menos por menos es más? In: GÓMEZ, P.; RICO, L. (Ed.). Iniciación a la investigación en didáctica de la matemática. Homenaje al profesor Mauricio Castro. Granada: Universidad de Granada, 2001. p. 257-275.

GUTIERREZ, J. Arte Breve y muy provechoso de quenta castellana y Arithmetica. Zaragoza: casa de Pedro Bernuz, 1564. 
JAHNKE, H. N. Cantor's cardinal and ordinal infinites: An epistemological and didactic view.

Educational Studies in Mathematics, Netherlands, v. 48, p. 175-197, 2001.

LÓPEZ, J. M. Ciencia y Técnica en la sociedad española de los siglos XVI y XVII. 1. ed. Barcelona: Labor, 1979. 511 p.

MADRID, M.J.; MAZ-MACHADO, A. Analysis of two Spanish arithmetic books written in the XVIcentury. Journal of Education, Psychology and Social Sciences, Zilina, v. 3, n. 2, p. 117-21, 2015.

MADRID, M.J.; MAZ-MACHADO, A.; LÓPEZ, C. Fenomenología y representaciones en el Dorado Contador de Miguel Gerónimo de Santa Cruz. ENSAYOS, Revista de la Facultad de Educación de Albacete, Albacete, v. 30, n. 1, p. 63-72, 2015.

MALET, A. Mil años de matemáticas en Iberia. In: DURÁN, A. J. El legado de las matemáticas: de Euclides a Newton: los genios a través de sus libros. Sevilla: Consejería de cultura (Junta de Andalucía), Universidad de Sevilla, Real Sociedad Matemática Española, SAEM Thales, 2000. p. 193224.

MAZ, A. Investigación histórica de conceptos en los libros de matemáticas. In: GONZÁLEZ, M.J.; GONZÁLEZ, M.T.; MURILLO, J. (Ed.). Investigación en Educación Matemática XIII. Santander: SEIEM, 2009. p. 5-20.

MAZ, A.; RICO, L. Negative numbers in the 18th and 19th centuries: phenomenology and representations. Electronic Journal of Research in Educational Psychology, Almeria, v. 17, n. 1, p. 537-554, 2009.

MAZ-MACHADO, A.; RICO, L. El Tratado elemental de matemáticas de José Mariano Vallejo en el bicentenario de su publicación. Suma: revista sobre la enseñanza y el aprendizaje de las matemáticas, Badalona, v. 74, p. 55-63, 2013.

MAZ-MACHADO, A.; RICO, L. Principios didácticos en textos españoles de matemáticas en los siglos XVIII y XIX. Revista Latinoamericana de Investigación en Matemática Educativa, México, v. 18, n. 1, p.49-76, 2015.

MAZ-MACHADO, A.; LÓPEZ, C.; SIERRA, M. Fenomenología y representaciones en la Arithmetica de Juan de Yciar. In: RICO, L et al. (Ed.). Investigación en Didáctica de las Matemática. Homenaje a Encarnación Castro. Granada: Editorial Comares, 2013. p. 77-84.

MEAVILLA, V.; OLLER, A. Gaspar de Texeda y los algoritmos de la multiplicación. Suma: revista sobre la enseñanza y el aprendizaje de las matemáticas, Badalona, v. 75, p. 61-73, 2014.

NATIONAL COUNCIL OF TEACHER OF MATHEMATICS. Curriculum and Evaluation Standars for School Mathematics. 1. ed. Reston VA: The Council, 1989. 258 p.

ORTEGA, J. Conpusicion de la arte de la arismetica y Juntamente de geometria. 1. ed. Lyon: casa de Maistro Nicolau de Benedictis, 1512. 203 p.

PARADÍS, J.; MALET, A. Los orígenes del álgebra: de los árabes al renacimiento. 1. ed. Barcelona: PPU, 1989. $261 \mathrm{p}$.

PÉREZ, J. La sociedad española del Renacimiento. 2000. Disponible en: <http://www.cervantesvirtual.com/bib/historia/CarlosV/6_2_josep_perez.shtml>. Acceso en: 24, marzo. 2017. 
PÉREZ DE MOYA, J. Arithmetica practica, y specvlativa. 1. Ed. Salamanca: Mathias Gast, 1562, $765 \mathrm{p}$.

PICADO, M.; RICO, L.; GÓMEZ, B. El sistema métrico decimal en textos de matemáticas para la instrucción primaria en las Islas Canarias en el siglo XIX. Números, La Laguna, v. 82, p. 37-53, 2013.

PICATOSTE, F. Apuntes para una biblioteca científica española del siglo XVI: estudios biográficos y bibliográficos de ciencias exactas, físicas y naturales y sus inmediatas aplicaciones en dicho siglo. 1. ed. Madrid: Manuel Tello, 1891, 416 p.

REY, J. Los matemáticos españoles del siglo XVI (No. 1). 1. ed. Madrid: Junta de Investigaciones Histórico-Bibliográficas, 1934. 162 p.

ROCHA, A. Arithmetica. 1. ed. Barcelona: casa de Claudio Bornat a la Águila Fuerte, 1564.

SALAVERT, V. Introducción a la historia de la aritmética práctica en la Corona de Aragón en el siglo XVI. Dynamis: Acta Hispanica Ad Medicinae Scientiarumque. Historiam Illustrandam, Granada, v. 10, p. 63-91. 1990.

SALAVERT, V. Aritmética y sociedad en la España del siglo XVI. In: GARMA. S.; FLAMENT, D.; NAVARRO, V. (Ed.). Contra los titanes de la rutina (contre les titans de la routine). Encuentro en Madrid de investigadores hispano-franceses sobre la historia y la filosofía de la matemática. Madrid: Consejo Superior de Investigaciones Científicas, 1994. p. 51-69.

SANTA CRUZ, M.G. Libro de arithmetica especvlativa, y práctica, intitvlado, el Dorado Contador, contiene la fineza y reglas de contar oro y plata, y los Aneajes de Flandes. Madrid: Viuda de Alonso Martín, 1625. 238 p.

SCHUBRING, G. On the methodology of analysing historical textbooks: Lacroix as textbook author. For the learning of mathematics, Fredericton, v. 7, n. 3, p. 41-51, 1987.

SCHUBRING, G. Discussions épistémologiques sur le statut des nombres négatifs et leur représentation dans les manuels allemands et français de mathématique entre 1795 et 1845 . In: LABORDE, C. (Ed.). Actes du premier colloque franco-allemand de didáctique des mathématiques et de l'informatique. La Pensée Sauvage: Grenoble, 1988. p. 137-145.

SEGOVIA, I.; RICO, L. Unidades didácticas. Organizadores. In: CASTRO, E. (Ed.). Didáctica de las matemáticas en la educación primaria. Madrid: Síntesis, 2001. p. 83-104.

SIERRA, M.; GONZÁLEZ, M. T.; LÓPEZ, C. Evolución histórica del concepto de límite funcional en los libros de texto de bachillerato y curso de orientación universitaria, 1940-1995. Enseñanza De Las Ciencias, Cerdanyola del Vallès, v. 17, n. 3, p. 463-476, 1999.

SIERRA, M., GONZÁLEZ, M. T. Y LÓPEZ, C. El concepto de continuidad en los manuales españoles de enseñanza secundaria de la segunda mitad del siglo XX. Educación Matemática, México, v. 15, n. 1, p. 21-50, 2003.

SIERRA, M.; RICO, L.; GÓMEZ, B. El número y la forma. Libros e impresos para la enseñanza del cálculo y la geometría. In: ESCOLANO, A. (Ed.). Historia Ilustrada de libro escolar en España. Del Antiguo Régimen a la Segunda República. Fundación Germán Sánchez Rupérez, 1997. p. 373398.

SMITH, D. E. Rara arithmetica. 1. ed. Boston: Ginn \& Company, 1908. 507 p.

TEXEDA, G. Suma de Arihtmetica Practica y de todas Mercaderias con la horden de contadores. 
1. ed. Valladolid: Oficina de Francisco Fernández de Córdoba, 1546. 64 p.

YCIAR, J. Arithmetica Practica. 1. ed. Zaragoza: casa de Pedro Bernuz, 1549. 56 p.

Submetido em 7 de Maio de 2016. Aprovado em 9 de Março de 2017. 